\title{
Principal component analysis-based inversion of effective temperatures for late-type stars ${ }^{\star}$ (Research Note)
}

\author{
F. Paletou ${ }^{1,2}$, M. Gebran ${ }^{3}$, E. R. Houdebine ${ }^{1,2,4}$, and V. Watson ${ }^{1,2}$ \\ ${ }^{1}$ Université de Toulouse, UPS-Observatoire Midi-Pyrénées, Irap, 31028 Toulouse Cedex 4, France \\ e-mail: fpaletou@irap.omp.eu \\ 2 CNRS, Institut de Recherche en Astrophysique et Planétologie, 14 Av. E. Belin, 31400 Toulouse, France \\ 3 Department of Physics and Astronomy, Notre Dame University-Louaize, PO Box 72, Zouk Mikaël, Lebanon \\ 4 Armagh Observatory, College Hill, BT61 9DG Armagh, Northern Ireland
}

Received 24 June 2015 / Accepted 14 July 2015

\begin{abstract}
We show how the range of application of the principal component analysis-based inversion method of Paletou et al. (2015, A\&A, 573, A67) can be extended to data for late-type stars. Besides being an extension of its original application domain, which applied to FGK stars, we also used synthetic spectra for our learning database. We discuss our results for effective temperatures in comparison with previous evaluations made available from VizieR and Simbad services at CDS.
\end{abstract}

Key words. virtual observatory tools - stars: fundamental parameters - stars: late-type

\section{Introduction}

Effective temperatures of late-type stars were inverted from HARPS spectra, using the principal component analysis-based (PCA) method detailed in Paletou et al. (2015). In the latter study, fundamental parameters of FGK stars were inverted using a so-called learning database generated from the Elodie stellar spectra library (see Prugniel et al. 2007) using observed spectra for which fundamental parameters were already evaluated. Also, spectra considered in the Paletou et al. (2015) study had typical spectral resolution $\mathcal{R}$ of 50000 (Allende Prieto el al. 2004) and 65000 (Petit et al. 2014), i.e. values significantly lower than HARPS data.

In this study, the inversion of the effective temperature, $T_{\text {eff }}$, from spectra of late-type (dwarf) stars of $\mathrm{K}$ and $\mathrm{M}$ spectral types is performed using a database of synthetic spectra. We discuss hereafter comparisons with published values collected from the VizieR and Simbad services of CDS.

\section{The learning database}

A grid of 6336 spectra was computed using SYNSPEC-48 synthetic spectra code (Hubeny \& Lanz 1992) and Kurucz ATLAS-12 model atmospheres (Kurucz 2005). The linelist was built from Kurucz (1992) gfhyperall . dat ${ }^{1}$.

For our purposes, we adopted a grid of parameters such that $T_{\text {eff }}$ is in a $3500-4600 \mathrm{~K}$ range with a $100 \mathrm{~K}$ step, $\log g$ is in the range of $4-5$ dex with a 0.2 dex step, metallicity $[\mathrm{Fe} / \mathrm{H}]$ is in a $-2-+0.5$ range with a 0.25 dex step and, finally, $v \sin i$ varies from 0 to $14 \mathrm{~km} \mathrm{~s}^{-1}$ with a $2 \mathrm{~km} \mathrm{~s}^{-1}$ step.

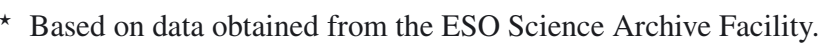

1 http://kurucz.harvard.edu
}

For all models, the microturbulent velocity was fixed at $\xi_{\mathrm{t}}=1 \mathrm{~km} \mathrm{~s}^{-1}$ and $[\alpha / \mathrm{Fe}]$ was set to 0 . The spectral resolution of the HARPS spectrograph, i.e. $\mathcal{R}=115000$ was adopted for the production of this set of synthetic spectra. We finally limited the study to a spectral band centred around the Na I D-doublet, ranging from 585.3 to $593.2 \mathrm{~nm}$.

Even though the choice of Atlas for such cool stars as well as the choice of the spectral bands we considered may be questionable, we show that effective temperatures we inverted from HARPS data are realistic enough for several further studies (e.g. rotation-activity correlations vs. the spectral type).

\section{Observational and reference data}

We used 57 high-resolution HARPS spectra taken from the ESO Science Archive Facility. The selection targeted late-type dwarf $\mathrm{K}$ stars, and early-type dwarf M stars. We also gave preference to high signal-to-noise spectra. Related objects are listed in Fig. 1; see also Table 1.

All reference data were collected from VizieR catalogues, using the ASTROQUERY ${ }^{2}$ Python modules already mentioned by Paletou \& Zolotukhin (2014).

\section{Results}

Given the "bulk" of nearest neighbours we consider for our inversion procedure, we estimate that $150 \mathrm{~K}$ is a typical upper value for the uncertainty in our derived effective temperature.

2 astroquery.readthedocs.org 


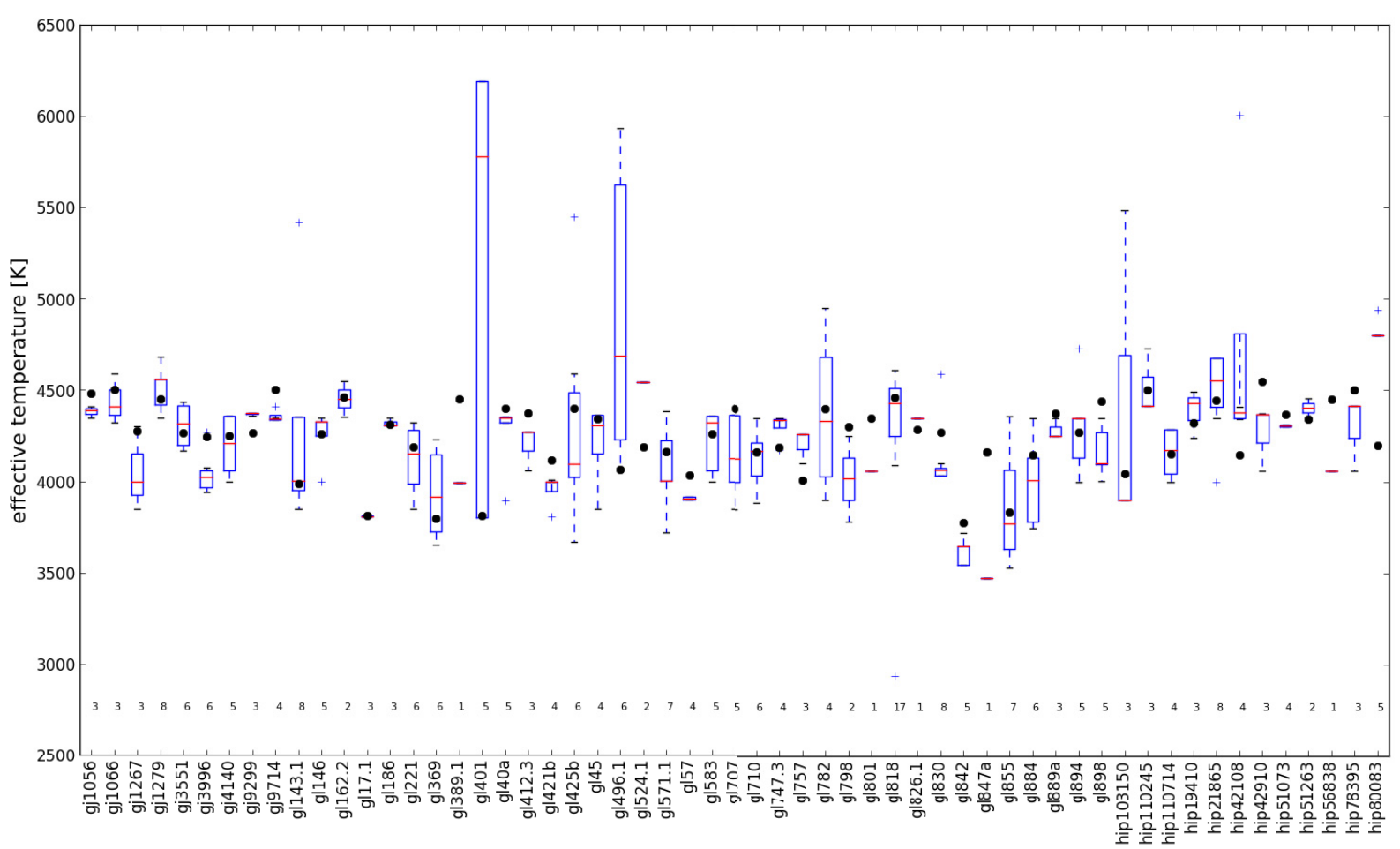

Fig. 1. Comparison between our estimate of effective temperatures $(\bullet)$, and the values we got from available VizieR catalogues. The latter collections are represented as classical boxplots. (The horizontal bar inside each box indicates the median, or $Q_{2}$ value, while each box extends from first quartile, $Q_{1}$, to third quartile $Q_{3}$. Extreme values, still within a 1.5 times the interquartile range away from either $Q_{1}$ or $Q_{3}$, are connected to the box with dashed lines. Outliers are denoted by a "+" symbol.) Objects we studied are listed along the horizontal axis. In addition, for each object at level $T_{\text {eff }} \sim 2800 \mathrm{~K}$, we explicated the number of values found among all VizieR catalogues.

From Fig. 1, we can identify eight major outliers considering deviations from our estimate of the effective temperature and values published and made available through the VizieR service at CDS.

GL 401 is a M3V star. It is also an interesting case, from the point of view of fundamental parameters made available. We retrieved five determinations of its effective temperature, spanning an impressive range of values, as can be seen from the corresponding boxplot in Fig 1. However, our estimate of $3810 \mathrm{~K}$ is in excellent agreement with the recent value of $3804 \mathrm{~K}$ derived by Gaidos et al. (2014).

The case of the K9V star GL 496.1 is very similar. We extracted six values from VizieR, and again, the most recent value of $4075 \mathrm{~K}$ given by Gaidos et al. (2014) is in excellent agreement with our estimate of $4063 \mathrm{~K}$. We also note the very different value of $4685 \mathrm{~K}$ given by Santos et al. (2013), and reported at Simbad at CDS for this object.

GL 389.1 is believed to be a K5.5V star (Gray et al. 2006). We could only identify only one data point at VizieR, an estimate of $3990 \mathrm{~K}$ given by Lafrasse et al. (2010, also catalogue id. II/320). Our own estimate is significantly hotter at $4450 \mathrm{~K}$, which appears to be more consistent with the spectral type found at Simbad.

According to Simbad at CDS, GL 847 A is a K4 star (Van Leeuwen 2007). This is clearly not consistent with our estimate of an effective temperature of $4160 \mathrm{~K}$, nor with the only value we could retrieve from VizieR, i.e. the estimate by Morales et al. (2008) yielding $3470 \mathrm{~K}$. As for the precedent object, the lack of data available in catalogues makes the assessment of a reliable reference value difficult.

HIP 42108 is a K6V star (Gray et al. 2006) for which we could estimate an effective temperature of 4147 K. From available catalogues, we found the most recent estimate of $4343 \mathrm{~K}$ given by McDonald et al. (2012), which is also very close to the alternative estimate of Wright et al. (2003). Lafrasse et al. (2010) also report a very close estimate of $4410 \mathrm{~K}$. For this object, however, Ammons et al. (2006) report a very different value of $6005 \mathrm{~K}$.

A similar case of overestimation has been identified for GL 524.1. We obtained an effective temperature of $4186 \mathrm{~K}$, while Ammons et al. (2006) provide a significantly larger value of $4554 \mathrm{~K}$. Unfortunately, we could not find alternative estimates for this parameter for this object (there is an entry for GL 524.1 reported by Morales et al. 2008, but no $T_{\text {eff }}$ value is given).

HIP 56838 is, according to Simbad, a K6V star based on the classification of Gray et al. (2006). Our estimate of its effective temperature is $4200 \mathrm{~K}$, in agreement with a K6 (main sequence) spectral type, while the only VizieR data we could get is $4060 \mathrm{~K}$ (Wright et al. 2003). Even though this object can also be found in the Ammons et al. (2006) catalogue, no $T_{\text {eff }}$ value is given there. Besides, a much cooler temperature of $3800 \mathrm{~K}$, which would better correspond to a M0V spectral type, was recently given by Kordopatis et al. (2013).

HIP 80083 is quite consistently given at $4800 \mathrm{~K}$ (Sousa et al. 2011; Adibekyan et al. 2012; Carretta 2013), or even hotter (Ammons et al. 2006). Our inversion procedure gives an effective temperature significantly lower at $4200 \mathrm{~K}$, typical of a spectral type later than K4, as indicated by Simbad at CDS.

Table 1 summarizes our results. It displays our inverted $T_{\mathrm{eff}}^{\text {(inv.) }}$ and two reference values. The first one, $T_{\text {eff }}^{\text {(clos.) }}$, was defined as the value found in VizieR catalogues closest to our estimate, while $T_{\mathrm{eff}}^{(\text {med.) }}$ is the median of all catalogue values. 
Table 1. Inverted and reference effective temperatures for all objects.

\begin{tabular}{|c|c|c|c|}
\hline Object & $T_{\text {eff }}^{\text {(inv.) }}[\mathrm{K}]$ & $T_{\mathrm{eff}}^{\text {(clos.) }}[\mathrm{K}]$ & $T_{\text {eff }}^{\text {(med.) }}[\mathrm{K}]$ \\
\hline gj1056 & 4480.0 & 4410.0 & 4391.0 \\
\hline gj1066 & 4500.0 & 4590.0 & 4410.0 \\
\hline gj1267 & 4275.0 & 4301.0 & 4000.0 \\
\hline gj1279 & 4450.0 & 4424.0 & 4556.0 \\
\hline gj3551 & 4267.0 & 4287.0 & 4318.5 \\
\hline gj3996 & 4244.0 & 4272.0 & 4023.0 \\
\hline gj4140 & 4250.0 & 4210.0 & 4210.0 \\
\hline gj9299 & 4267.0 & 4360.0 & 4372.0 \\
\hline gj9714 & 4500.0 & 4410.0 & 4344.5 \\
\hline gl143.1 & 3985.0 & 3970.0 & 4001.0 \\
\hline g1146 & 4260.0 & 4250.0 & 4329.0 \\
\hline gl162.2 & 4462.5 & 4550.0 & 4452.5 \\
\hline gl17.1 & 3812.5 & 3809.0 & 3809.0 \\
\hline gl186 & 4311.0 & 4307.0 & 4307.0 \\
\hline gl221 & 4186.0 & 4282.0 & 4151.0 \\
\hline g1369 & 3796.0 & 3915.0 & 3915.0 \\
\hline gl389.1 & 4450.0 & 3990.0 & 3990.0 \\
\hline g1401 & 3810.0 & 3804.0 & 5780.0 \\
\hline gl40a & 4400.0 & 4352.0 & 4350.0 \\
\hline g1412.3 & 4375.0 & 4271.0 & 4271.0 \\
\hline g1421b & 4117.0 & 4008.0 & 3995.0 \\
\hline g1425b & 4400.0 & 4590.0 & 4097.5 \\
\hline g145 & 4340.0 & 4361.0 & 4305.5 \\
\hline g1496.1 & 4063.0 & 4075.0 & 4685.0 \\
\hline gl524.1 & 4186.0 & 4544.0 & 4544.0 \\
\hline gl571.1 & 4164.0 & 4060.0 & 4002.0 \\
\hline g157 & 4033.0 & 3913.0 & 3906.5 \\
\hline gl583 & 4261.0 & 4320.0 & 4320.0 \\
\hline g1707 & 4400.0 & 4364.0 & 4125.0 \\
\hline g1710 & 4160.0 & 4130.0 & 4165.0 \\
\hline g1747.3 & 4186.0 & 4170.0 & 4337.0 \\
\hline g1757 & 4008.0 & 4100.0 & 4259.0 \\
\hline g1782 & 4400.0 & 4590.0 & 4330.0 \\
\hline g1798 & 4300.0 & 4250.0 & 4015.5 \\
\hline g1801 & 4350.0 & 4060.0 & 4060.0 \\
\hline g1818 & 4462.5 & 4444.0 & 4430.0 \\
\hline g1826.1 & 4287.5 & 4350.0 & 4350.0 \\
\hline $\mathrm{g} 1830$ & 4269.0 & 4100.0 & 4065.5 \\
\hline g1842 & 3776.0 & 3720.0 & 3649.0 \\
\hline g1847a & 4160.0 & 3470.0 & 3470.0 \\
\hline g1855 & 3831.0 & 3771.0 & 3771.0 \\
\hline $\mathrm{g} 1884$ & 4147.0 & 4130.0 & 4009.5 \\
\hline g1889a & 4371.0 & 4350.0 & 4251.0 \\
\hline g1894 & 4270.0 & 4350.0 & 4350.0 \\
\hline g1898 & 4440.0 & 4350.0 & 4101.0 \\
\hline hip103150 & 4043.0 & 3900.0 & 3900.0 \\
\hline hip110245 & 4500.0 & 4416.0 & 4416.0 \\
\hline hip110714 & 4150.0 & 4060.0 & 4172.0 \\
\hline hip19410 & 4320.0 & 4238.0 & 4432.0 \\
\hline hip21865 & 4445.5 & 4432.0 & 4555.0 \\
\hline hip42108 & 4147.0 & 4343.0 & 4380.0 \\
\hline hip42910 & 4550.0 & 4373.0 & 4368.0 \\
\hline hip51073 & 4367.0 & 4310.0 & 4305.0 \\
\hline hip51263 & 4343.0 & 4350.0 & 4403.5 \\
\hline hip56838 & 4450.0 & 4060.0 & 4060.0 \\
\hline hip78395 & 4500.0 & 4414.0 & 4414.0 \\
\hline hip80083 & 4200.0 & 4800.0 & 4800.0 \\
\hline
\end{tabular}

Notes. Hereafter $T_{\text {eff }}^{\text {(clos.) }}$ was defined as the value found in VizieR catalogues closest to our inverted $T_{\text {eff }}^{\text {(inv.) }}$, while $T_{\text {eff }}^{\text {(med.) }}$ is the median of catalogues values.
Finally, to characterize our results, we first removed the eight above mentioned outliers from our objects list, which is about $14 \%$ of the original sample. Considering reference values as the one closest to our inverted effective temperature, we obtain a (mean signed difference or) bias of $21 \mathrm{~K}$ and a standard deviation of $90 \mathrm{~K}$. If we use the median value as reference, the bias is $60 \mathrm{~K}$ and the standard deviation $132 \mathrm{~K}$.

\section{Conclusion}

We have shown that the PCA-based inversion method of Paletou et al. (2015) provides realistic values for the effective temperature of late-type stars. Comparisons made between our estimates and effective temperature data found in the available literature reveals the existence of some strong discrepancies for a few objects. These discrepancies are most often related to very limited samples of estimates, so that additional investigations are clearly required for these objects.

These should consist in using different synthetic spectra, which can produce other radiative modelling tools such as Marcs (Gustafsson et al. 2008) or Phoenix, for cool stars (see e.g. Husser et al. 2013). The consideration of other spectral domains, and eventually the use of a combination of several distinct spectral domains should be explored too.

Our study also raises the more general question of the consistency between published (and made available) data, as well as the consistency between data provided by VizieR and Simbad services.

Acknowledgements. This research has made use of the VizieR catalogue access tool, CDS, Strasbourg, France. The original description of the VizieR service was published in A\&AS 143, 23. This research has made use of the SIMBAD database, operated at CDS, Strasbourg, France.

\section{References}

Adibekyan, V. Zh., Sousa, S. G., Santos, N. C., et al. 2012, A\&A, 545, A32 Allende Prieto, C., Barklem, P. S., Lambert, D. L., \& Cunha, K. 2004, A\&A, 420, 183

Ammons, S. M., Robinson, S. E., Strader, J. et al. 2006, ApJ, 638, 1004 Carretta, E. 2013, A\&A, 557, A128

Gaidos, E., Mann, A. W., Lépine, S., et al. 2014, MNRAS, 443, 2561

Gray, R. O., Corbally, C. J., Garrison, R. F., et al. 2006, AJ, 132, 161

Gustafsson, B., Edvardsson, B., Eriksson, K., et al. 2008, A\&A, 486, 951 Hubeny, I., \& Lanz, T. 1992, A\&A, 262, 501

Husser, T.-O., Wende-von Berg, S., Dreizler, S., et al. 2013, A\&A, 553, A6

Kordopatis, G., Gilmore, G., Steinmetz, M., et al. 2013, AJ, 146, 134

Kurucz, R. L. 1992, Rev. Mex. Astron. Astrofis., 23, 45

Kurucz, R. L. 2005, Mem. Soc. Astron. It. Supp., 8, 14

Lafrasse, S., Mella, G., Bonneau, D., et al. 2010, SPIE Conf. Astronomical Instrumentation, $77344 \mathrm{E}, 140$

McDonald, I., Zijlstra, A. A., \& Boyer, M. L. 2012, MNRAS, 427, 343

Morales, J. C., Ribas, I., \& Jordi, C. 2008, A\&A, 478, 507

Paletou, F., \& Zolotukhin, I. 2014, ArXiv e-prints [arXiv: 1408. 7026]

Paletou, F., Böhm, T., Watson, V., \& Trouilhet, J.-F. 2015, A\&A, 573, A67

Petit, P., Louge, T., Théado, S., et al. 2014, PASP, 126, 469

Prugniel, P., Soubiran, C., Koleva, M., \& Le Borgne, D. 2007, ArXiv e-prints [arXiv: astro-ph/0703658]

Santos, N. C., Sousa, S. G., Mortier, A., et al. 2013, A\&A, 556, A150

Sousa, S. G., Santos, N. C., Israelian, G., Mayor, M., \& Udry, S. 2011, A\&A, 533, A141

van Leeuwen, F. 2007, A\&A, 474, 653

Wright, C. O., Egan, M. P., Kraemer, K. E., \& Price, S. D. 2003, AJ, 125, 359 